are efficacious with a single dose," says Leah Lipsich, vice president of strategic program direction at Regeneron. It was initially designed as a three-shot product, but nonhuman primate experiments demonstrated a protective effect after two doses. The company then found that a higher front-end dose provided similar protection, even in symptomatic animals (J. Infect. Dis. doi:10.1093/infdis/ jiy285, 2018). The Vaccine Research Center at the National Institute of Allergy and Infectious Diseases and partners are working on another single-shot therapy. Mab114, a single-antibody drug, was identified by screening the memory B-cell repertoire of an Ebola virus infection survivor from a 1995 outbreak in the DRC. It protected macaque monkeys when given five days after an Ebola challenge (Science 351, 1339-1342, 2016). Mab114 is currently low on the WHO priority list, however, due to the paucity of clinical data. So, too, is favipiravir, developed by Fujifilm-owned Toyama Chemical. Its efficacy is uncertain, and the appropriate dose has not yet been established, according to the WHO's own assessment

Of course, with an agent such as Ebola, human clinical trials can only evaluate a drug's safety, tolerability and pharmacokinetic profile. To assess efficacy, drug developers must conduct non-human primate challenge studies and this requires access to BSL- 4 facilities, which are limited in number. It's a major bottleneck for drug developers. "Data is very hard to come by because it's very hard to do these studies," says Christos Kyratsous, senior director for infectious diseases and viral vector technologies at Regeneron. "Everybody is competing for the same space."

The lack of commercial viability for antiEbola agents is another major drag on development. Some of the programs that started to scale up just as the first major crisis started to abate had built enough momentum to continue into the present time, but others have fallen by the wayside. Arbutus Biopharma, of Vancouver, British Columbia, shelved a promising lipidnanoparticle-formulated short interfering RNA drug TKM-130803. Novavax parked an adjuvanted lipid-nanoparticle-based recombinant vaccine based on Ebola GP trimers, despite what Gregory Glenn, its president of R\&D, describes as promising data and a clear path to licensure. "There is no market," he says. "Kudos to Merck for being able to carry on with development, when there's very little opportunity there." Last September, the US Biomedical Advanced Research and Development Authority (BARDA) earmarked four programs for development funding and stockpile purchases: vaccine programs from Johnson \& Johnson and Merck, which received $\$ 44.7$ million and $\$ 39.2$ million, respectively, and MappBio's ZMapp and Regeneron's REGN-EB3 programs, which received $\$ 45.9$ million and $\$ 40.4$ million, respec- tively. Without a similar guarantee, Novavax was unable to move forward.

It's still an open question whether the resources that have been brought to bear on the present outbreak have been enough. The West African outbreak, which led to 28,616 cases and 11,310 deaths, was unprecedented in its scale and duration. Each of the eight Ebola outbreaks the DRC experienced between 1976 and 2017 involved fewer than 400 cases (some far fewer than that) and resolved within six months. These smaller outbreaks may offer a better benchmark against which to gauge the effectiveness of the Merck vaccine in limiting the current outbreak. The hope is that the health authorities' capabilities-and those of others tackling future outbreaks-will be strengthened by the addition of effective vaccines and drugs, but the management of the response will always remain critical. "If you look at the response now compared with 2014, it's just night and day," says Richard Hatchett, the former deputy director and chief medical officer at BARDA, who is now the CEO of the London-based Coalition for Epidemic Preparedness Innovations, a multilateral body that has raised $\$ 620$ million of its $\$ 1$ billion target to accelerate the development of vaccines against emerging pathogens. The current response may offer a blueprint for future outbreaks. "This will be seen as a pivotal moment."

Cormac Sheridan Dublin

\title{
Saying aye to the artificial iris
}

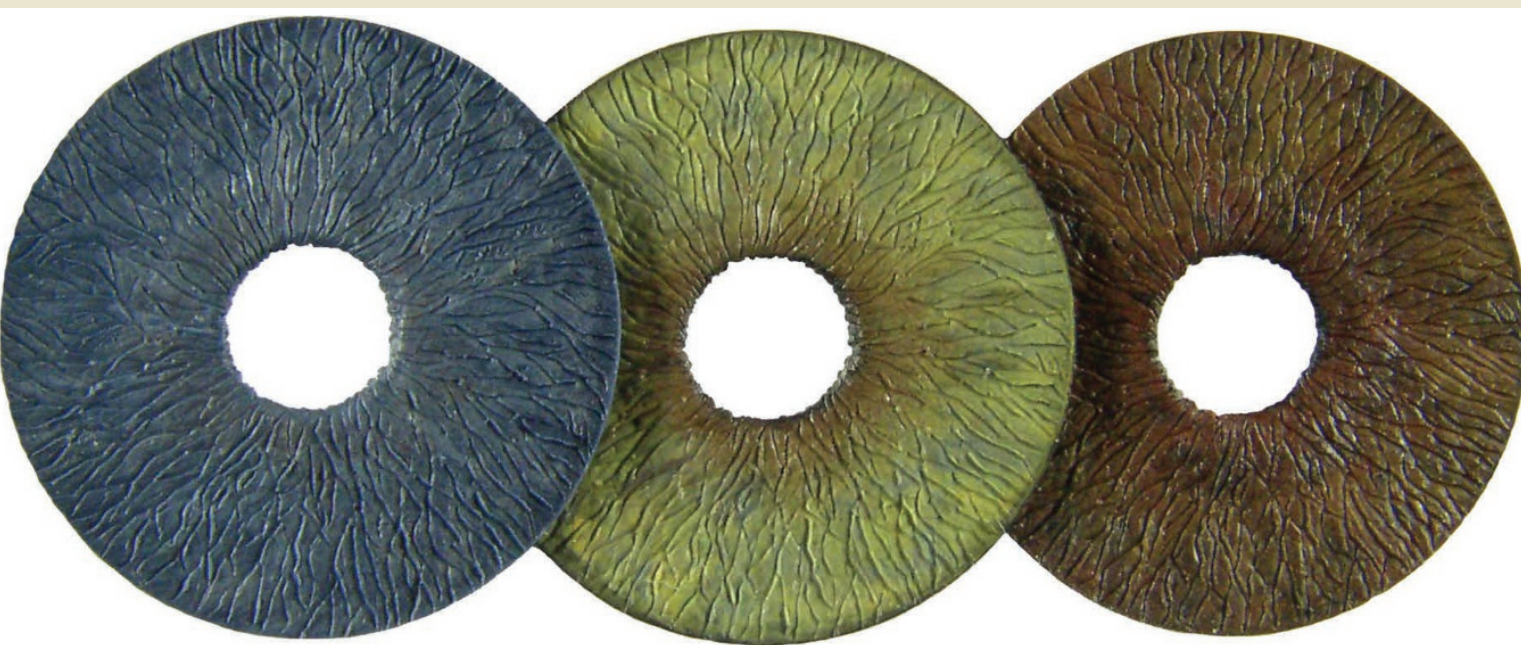

The US Food and Drug Administration (FDA) has greenlit the first artificial iris in the US - a surgically implanted device to treat patients with missing or damaged irises due to a congenital condition called aniridia or other damage to the eye, such as albinism, traumatic injury or surgical removal due to melanoma. Congenital aniridia is a rare genetic disorder affecting up to 1 in 100,000 people in the US, and those with the condition have sensitivity to light and other severe vision problems, as the iris controls the amount of light entering the eye. The CustomFlex Artificial Iris was developed by Germany-based HumanOptics, It is made of thin, foldable medical-grade silicone and is custom-fitted and colored for each individual patient. The artificial iris was granted breakthrough device designation, meaning the FDA provided extensive guidance to the company on the device's development to expedite evidence generation and the agency's review. 